\title{
Analysis of Gaussian-beam degenerate optical parametric amplifiers for the generation of quadrature-squeezed states
}

\author{
Kahraman G. Köprülü and Orhan Aytür \\ Department of Electrical and Electronics Engineering, Bilkent University, TR-06533 Bilkent, Ankara, Turkey
}

(Received 2 March 1999)

\begin{abstract}
This paper investigates the generation of quadrature-squeezed states of light using a degenerate optical parametric amplifier (DOPA) that is pumped by a focused Gaussian beam. The formulation that is presented facilitates the calculation of squeezing for an arbitrary local oscillator beam. This formulation also establishes a formal equivalence between the classical parametric gain and the measured level of squeezing. The maximum squeezing that can be achieved using a Gaussian-beam local oscillator is determined to be limited to 13.4 $\mathrm{dB}$, as a consequence of gain-induced diffraction. The phase lag of the maximally squeezed quadrature is shown to be significantly different from the plane-wave theoretic value of $\pi / 2$, unless the focusing is very weak. The use of a second DOPA for generating a local oscillator beam that is matched to the squeezed field is also investigated. In this case, squeezing is limited only by the available pump power.
\end{abstract}

[S1050-2947(99)09911-4]

PACS number(s): 42.65.Yj, 42.50.Dv, 42.50.Lc

\section{INTRODUCTION}

Degenerate optical parametric amplifiers (DOPA's) play an important role in the generation of nonclassical states of light such as squeezed states [1-4]. A DOPA is a phasesensitive light amplifier, whose gain depends on the optical phase difference between the pump and the input signal fields. This property results in one phase quadrature of the input signal field being amplified (parametric gain greater than unity), while the orthogonal quadrature gets deamplified (parametric gain less than unity). When the input signal to a DOPA is in a vacuum state, the output signal becomes a quadrature-squeezed vacuum state that exhibits quantum fluctuations that are below the quantum limit for the deamplified quadrature [2,5].

In this paper, we present an accurate and detailed analysis of Gaussian-beam pumped DOPA's. Modeling of practical DOPA's is crucial for both designing experiments aimed at generating large levels of squeezing, and also understanding the spatial properties of the generated squeezed field. In a typical experiment, the Gaussian-beam nature of the pump field brings about transverse gain variations that influence the behavior of the DOPA. A number of authors have investigated the effects of these gain variations in Gaussian-beam pumped DOPA's [6-8]. These models have shown that the signal field experiences phase and amplitude distortions that influence the net gain or squeezing achieved by the DOPA. In particular, the authors of Ref. [6] concluded that quadrature squeezing with a Gaussian-beam DOPA is limited to 6 $\mathrm{dB}$ as a consequence of these distortions. Even though this model brings out the importance of phase and amplitude distortions in the signal field, its quantitative conclusions are incorrect as a consequence of two erroneous assumptions. First, the authors have assumed that the phase of the maximally squeezed quadrature lags the pump phase by $\pi / 2$, which is the value predicted by the plane-wave theory of DOPA's [9]. This false assumption leads to a significant underestimation of the squeezing level that can be achieved with a Gaussian-beam pumped DOPA. Second, this model neglects the Gouy phase of the pump beam, resulting in a miscalculation of the phase-sensitive gain at regions away from the focus. On the other hand, the classical analysis of Ref. [8] also assumes the plane-wave theoretic value of $\pi / 2$ for the phase lag of the maximally deamplified quadrature. Furthermore, this model is limited to the weak focusing regime, where the confocal parameter of the pump beam is much larger than the interaction length. Likewise, the model of Ref. [7] is also limited to the weak focusing regime, and takes the optimal phase lag to be $\pi / 2$.

The Gaussian-beam pumped DOPA model presented in this paper facilitates accurate calculation of the phasesensitive DOPA gain and the resulting squeezing. Our model is valid in all focusing regimes, as long as the parametric interaction can be considered to be perfectly phase matched. This analysis is based on a modal expansion of the signal field in terms of cylindrically symmetric Laguerre-Gaussian beams. In Sec. II, we outline our formulation for calculating the classical small-signal gain of a Gaussian-beam pumped DOPA for an arbitrary cylindrically symmetric input signal beam. In Sec. III, we investigate the generation of squeezed vacuum states with a DOPA, and the detection of these states using a local oscillator (LO) beam of arbitrary profile. Here we show that the measured squeezing level is equal to the classical deamplification (one over the parametric gain) experienced by an arbitrary input signal to the DOPA, if the conjugate of that input were to be used as the LO beam in the detection of squeezed vacuum generated by the same DOPA. An important consequence is that the classical formulation of Sec. II can be used to calculate squeezing. In Sec. IV, we apply the results of the previous sections to the case of a Gaussian beam LO (or input signal). Here, we show that the maximum squeezing that can be achieved with a Gaussian LO beam is about $13 \mathrm{~dB}$, a figure much higher than the $6-\mathrm{dB}$ limit of Ref. [6]. In Sec. V, we investigate the generation of LO beams that are matched to the distorted spatial profile of the squeezed field $[10,11]$. Here we show that a matched LO beam can be generated with a DOPA that is identical to the 
one used for the generation of squeezed vacuum, and that the squeezing level detected using such a matched LO is limited only by the available pump power.

\section{CLASSICAL DOPA ANALYSIS}

In this section, we formulate a method for calculating the classical small-signal gain of a traveling-wave DOPA pumped by a focused Gaussian beam. We consider a geometry where the waist of the pump beam is located at the center of the nonlinear crystal. We assume that there is perfect phase matching, no pump depletion, and no spatial walkoff between the pump and the signal beams. This formulation facilitates the calculation of the small-signal gain of a DOPA for any (cylindrically symmetric) input signal beam.

Since the configuration at hand has cylindrical symmetry, we express all fields as $+z$ propagating waves with transverse amplitude profiles that depend only on the radial distance $\rho=\sqrt{x^{2}+y^{2}}$ and the propagation distance $z$. For a pump beam at an optical frequency of $2 \omega$, we consider a signal field described by

$$
E_{s}(\mathbf{r}, t)=\frac{1}{2} A_{s}(\rho, z) \exp \left[i\left(\omega t-k_{s} z\right)\right]+\text { c.c. }
$$

where c.c. denotes the complex conjugate of the first term. Under the slowly varying envelope approximation, the signal field amplitude $A_{s}(\rho, z)$ is governed by [8]

$$
\frac{\partial A_{s}(\rho, z)}{\partial z}-\frac{1}{2 i k_{s}} \nabla_{\perp}^{2} A_{s}(\rho, z)=-i \kappa A_{p}(\rho, z) A_{s}^{*}(\rho, z),
$$

where $A_{p}(\rho, z)$ is the pump field amplitude, $\kappa=\omega d_{e} / n_{s} c$ is the nonlinear coupling constant, $d_{e}$ is the effective nonlinear coefficient, $n_{s}$ is the refractive index, $k_{s}=n_{s} \omega / c$ is the wave number of the signal field, and $\nabla_{\perp}^{2}=\partial^{2} / \partial \rho^{2}+(1 / \rho) \partial / \partial \rho$ is the transverse Laplacian in cylindrical coordinates.

Equation (2) applies to an optical parametric amplifier (OPA) that is degenerate in all aspects including the polarization direction; a type-I phase-matched DOPA satisfies this requirement. However, if the OPA is type-II phase matched with orthogonally polarized signal and idler modes, a combination of these modes has to be considered as the signal of the DOPA. In this case, $n_{s}$ in Eq. (2) becomes the geometric mean of the refractive indices of the signal and idler modes. However, since the wave numbers of the two modes are different, it is not possible to define a single $k_{s}$ value for the combined mode. For type-II phase-matched OPA's, Eq. (2) holds approximately if the wave numbers of the signal and idler modes are close to each other.

In a plane-wave analysis, the $\rho$ dependence of the fields and hence the transverse Laplacian term in Eq. (2) disappear. In this case, the pump field amplitude is a constant $A_{p}(z)$ $=\left|A_{p}\right| \exp \left(i \phi_{p}\right)$, and, since the interaction is phase matched, the pump wave number $k_{p}=2 k_{s}$. The solution of the planewave problem for a crystal of length $l$ that is centered at $z$ $=0$ is [9]

$$
A_{s}(l / 2)=\mu A_{s}(-l / 2)-i \exp \left(i \phi_{p}\right) \nu A_{s}^{*}(-l / 2),
$$

where

$$
\begin{gathered}
\mu=\cosh \left(\kappa l\left|A_{p}\right|\right), \\
\nu=\sinh \left(\kappa l\left|A_{p}\right|\right) .
\end{gathered}
$$

The DOPA gain is given by

$$
g(\theta)=\left|\frac{A_{s}(l / 2)}{A_{s}(-l / 2)}\right|^{2}=|\mu+\exp [i(\theta-\pi / 2)] \nu|^{2},
$$

where $\theta=\phi_{p}-2 \phi_{s}$ is the phase difference between the pump and the signal waves at the input of the crystal, and $A_{s}\left(-l / z_{0}\right)=\left|A_{s}\right| \exp \left(i \phi_{s}\right)$. Equation (6) reveals the phase dependent nature of the DOPA gain. The maximum amplification is

$$
\max \{g(\theta)\}=(\mu+\nu)^{2}=\exp \left(2 \kappa l\left|A_{p}\right|\right),
$$

and occurs at $\theta=\pi / 2$. On the other hand, maximum deamplification $\max \{1 / g(\theta)\}$ is equal to maximum amplification in magnitude, and occurs at $\theta=-\pi / 2$.

The field amplitude of a Gaussian pump beam can be written as

$$
A_{p}(\rho, z)=\frac{A_{p 0}}{1-2 i z / z_{0}} \exp \left(\frac{-\rho^{2} / W_{0}^{2}}{1-2 i z / z_{0}}\right)
$$

where $W_{0}$ is the radius of the beam waist located at $z=0$, $z_{0}=k_{p} W_{0}^{2}$ is the confocal parameter (twice the Rayleigh range), $k_{p}=2 k_{s}$ is the pump wave number, and $A_{p 0}$ $=\left|A_{p 0}\right| \exp \left(i \phi_{p}\right)$ is a complex constant whose amplitude is related to the pump power $P_{p}$ through $\left|A_{p 0}\right|$ $=\sqrt{8 \omega P_{p} / \pi z_{0} c^{2} \epsilon_{0}}$. The transformations $z=\xi z_{0}$ and $\rho$ $=r W_{0}$ can be used to normalize Eq. (2) as

$$
\frac{\partial A_{s}(r, \xi)}{\partial \xi}+i \nabla_{\perp}^{2} A_{s}(r, \xi)=-i \gamma \exp \left(i \phi_{p}\right) u_{p}(r, \xi) A_{s}^{*}(r, \xi)
$$

where $\phi_{p}$ is the phase of the pump beam, $\gamma=\kappa z_{0}\left|A_{p 0}\right|$ is a constant, and

$$
u_{p}(r, \xi)=\frac{1}{1-2 i \xi} \exp \left(\frac{-r^{2}}{1-2 i \xi}\right)
$$

Since the DOPA is a phase-sensitive amplifier, it is desirable to keep the phase difference $\theta=\phi_{p}-2 \phi_{s}$ constant at all points along both the transverse and longitudinal directions. For this reason, it is common practice to choose the input signal to be a Gaussian beam that has the same confocal parameter and beam waist location as the pump beam. Figure 1 shows the phase fronts of the signal and pump beams in the absence of any parametric interaction between them. Even though the phase curvatures of the two beams are the same everywhere, the presence of the Gouy phase terms results in a gradual slip in the phase difference $\theta$ as a function of $z$. This phase slip should not be neglected even for $l / z_{0} \ll 1$, since the derivative of the Gouy phase with respect to $z$ is maximum at $z=0$. The magnitude of the gain is influenced by this phase slip even at small values of $l / z_{0}$.

Note that in the Gaussian beam analysis of the DOPA, it is convenient to define $\theta$ as the phase difference between the pump and the signal at $z=0$ as if there is no interaction 


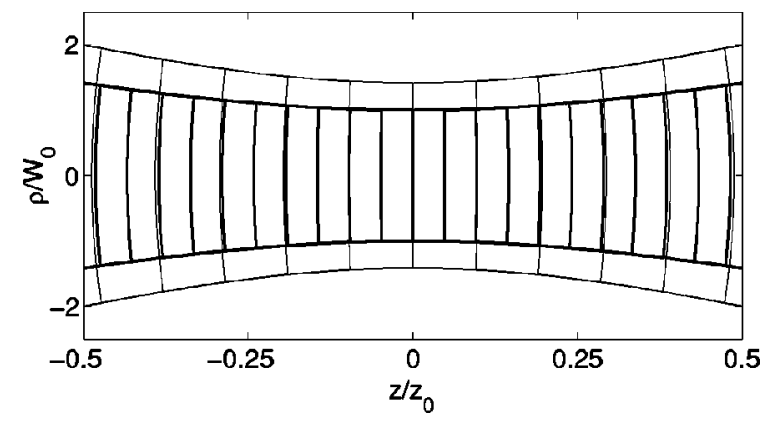

FIG. 1. Phase fronts of the Gaussian pump and signal beams inside the nonlinear crystal in the absence of the DOPA interaction. The bounding curves show the beam radii ( $1 / e^{2}$ of peak intensity). For clarity, the wavelength is exaggerated and the pump phase is shifted so that the phase fronts coincide at $z=0$.

between them. This convention assures that the definition of $\theta$ is independent of $l / z_{0}$. Alternatively, the phase difference can be defined at the input plane $(z=-l / 2)$. This phase difference $\theta^{\prime}$ is related to the one defined at the center through $\theta^{\prime}=\theta+\tan ^{-1}\left(l / z_{0}\right)$. However, we do not prefer this alternative definition, since $\theta^{\prime}$ depends on $l / z_{0}$ whereas $\theta$ does not.

In order to calculate the gain experienced by an arbitrary signal input beam, Eq. (9) has to be solved with the initial condition $A_{s}\left(r, \xi=-\xi_{0}\right)$, where $\xi_{0}=l / 2 z_{0}$. Equation (9) does not have an exact analytical solution even when the input signal is a Gaussian beam. An approximate analytical solution for a Gaussian input was introduced by Choi et al. [8]. In this analysis, the signal is assumed to have the same waist location and confocal parameter as the pump, and the solution is obtained using a perturbative method where the result is expressed in terms of powers of $l / z_{0}$ up to the quadratic term. This analysis has shown that the transverse profile of the pump intensity results in phase and amplitude distortions in the signal field, an effect known as gaininduced diffraction (GID). The overall effect of these distortions integrated in the transverse plane brings a limit to the maximum deamplification that can be obtained using a DOPA. However, the solutions in this formulation are only to the second order in $l / z_{0}$, and hence the validity of this model is limited to $l / z_{0} \ll 1$.

In another analysis introduced by La Porta and Slusher [6], the signal field is expressed as an infinite sum of orthogonal spatial modes, and the coupling between these modes as they propagate through the DOPA is investigated. However, this model contains some errors that have significant influence on the results and conclusions. Most importantly, the Gouy phase of the pump beam is missing in this analysis, leading to an error in the calculation of the squeezing level.

The two aforementioned models share an important misconception that deamplification (or squeezing) assumes its maximum value at a phase difference $\theta=-\pi / 2$, the value given by the plane-wave theory. In Sec. IV, we show that the $\theta$ value that maximizes deamplification depends on $l / z_{0}$, and may differ from $-\pi / 2$ significantly. Overlooking this issue results in an underestimation of the deamplification or squeezing levels by several $\mathrm{dB}$.

In our analysis, we express the signal field as a sum of orthonormal spatial modes, much like in the analysis of $\mathrm{La}$
Porta and Slusher. [The linearity of Eq. (9), which makes such an expansion possible, is a consequence of the undepleted pump approximation.] Using this expansion, we convert Eq. (9) into an ordinary differential equation in matrix form. This form is useful because it lends itself easily to accurate numerical calculations, and also yields valuable information on the coupling between the modes.

The transverse field variations in Eq. (9) may be handled with other numerical techniques as well. Finite differencing in the $r$ plane is another method that we have considered. However, this method has a few disadvantages. The step sizes in $z$ and $r$ must satisfy a stability condition, and hence cannot be chosen arbitrarily small. Furthermore, a finitesized window has to be used in the transverse plane, and even when absorbing boundary conditions are used, this introduces additional errors. Moreover, for larger $l / z_{0}$ values, the window size has to be increased to keep numerical errors small, and this increases the size of the equations. Finally, numerical errors due to finite differencing are larger than those of the mode expansion method.

Equation (9) reduces to the paraxial Helmholtz equation when its right-hand side is equal to zero. The general solution of this homogeneous equation can be written in terms of Laguerre-Gaussian beams. (The cylindrical symmetry of the geometry makes Laguerre-Gaussian beams more suitable than Hermite-Gaussian beams.) In general, LaguerreGaussian beams in cylindrical coordinates $(r, \varphi, \xi)$ have two indices, one associated with $r$ and the other with $\varphi$. Since the geometry at hand has cylindrical symmetry, we drop the second index and consider only the set of cylindrically symmetric modes. Therefore, the homogeneous solution can be written as

$$
A_{s}(r, \xi)=\sum_{n=0}^{\infty} A_{n} G_{n}(r, \xi)
$$

where $A_{n}$ is the complex amplitude of the $n$th cylindrically symmetric Laguerre-Gaussian mode,

$$
\begin{aligned}
G_{n}(r, \xi)= & L_{n}\left(\frac{r^{2}}{1+4 \xi^{2}}\right) \frac{1}{1-i 2 \xi} \\
& \times \exp \left(\frac{-r^{2} / 2}{1-i 2 \xi}\right) \exp \left(i 2 n \tan ^{-1} 2 \xi\right),
\end{aligned}
$$

$L_{n}$ being the $n$ th-order Laguerre polynomial. The cylindrically symmetric Laguerre-Gaussian beams are expressed in such a way that they all have the same confocal parameter as the pump, and their waists are all located at $\xi=0$. The beams defined in Eq. (12) form an orthonormal set, where

$$
2 \int_{0}^{\infty} G_{n}(r, \xi) G_{m}^{*}(r, \xi) r d r=\delta_{n m} .
$$

We use this orthonormal set to express the solution of the inhomogeneous equation by allowing the mode amplitudes to be functions of $\xi$. Substituting $A_{s}(r, \xi)$ into Eq. (9), multiplying both sides with $G_{m}^{*}$, and integrating over the transverse plane, we obtain, 


$$
\begin{aligned}
\frac{d}{d \xi} A_{m}(\xi)= & -i 2 \gamma \exp \left(i \phi_{p}\right) \\
& \times \sum_{n=0}^{\infty}\left[\int_{0}^{\infty} u_{p}(r, \xi) G_{m}^{*}(r, \xi) G_{n}^{*}(r, \xi) r d r\right] A_{n}^{*}(\xi) .
\end{aligned}
$$

Equation (14) relates the rate of change of the amplitude of the $m$ th mode to the amplitude of the $n$th mode. Equation (14) may be written in matrix form as

$$
\frac{d}{d \xi} A=T A^{*},
$$

where $A$ is the signal vector whose elements are the mode amplitudes $A_{n}(\xi)$, and $T$ is a square coefficient matrix whose elements are

$$
\begin{aligned}
T_{m n}= & -\left[\frac{2^{-(m+n+1)}(m+n) !}{m ! n !}\right]\left[\frac{i \gamma \exp \left(i \phi_{p}\right)}{\sqrt{1+4 \xi^{2}}}\right] \\
& \times \exp \left[-i(2 m+2 n+1) \tan ^{-1} 2 \xi\right],
\end{aligned}
$$

as a result of the integral in Eq. (14). Therefore, the partial differential equation of Eq. (9) is now reduced to an ordinary differential equation in matrix form.

The matrix element $T_{m n}$ represents the coupling between the $m$ th and the $n$th modes. The first factor in Eq. (16) shows how the amplitude of $T_{m n}$ depends on the mode indices. The fundamental mode at the input is coupled most strongly to the fundamental mode at the output, and the coupling decreases monotonically as the output mode index increases. The denominator in the second factor signifies the dependence of the coupling on the interaction distance $\xi$; the coupling between the modes decreases as $\xi$ increases, as a result of decreasing pump intensity away from the beam waist at $\xi=0$. The last factor is a consequence of the Gouy phases of the pump, input, and output modes. This factor is present even when $m=n=0$, resulting in a phase slip between the pump and the fundamental mode of the signal, as shown in Fig. 1.

The linearity of Eq. (15) allows us to write the general solution as

$$
A\left(\xi_{0}\right)=M A\left(-\xi_{0}\right)-i \exp \left(i \phi_{p}\right) N A *\left(-\xi_{0}\right),
$$

where $M\left(\gamma, \xi_{0}\right)$ and $N\left(\gamma, \xi_{0}\right)$ are state transition matrices that relate the output to the input signal field. In Appendix A, we show that $M$ and $N$ are symmetric and Hermitian matrices, respectively. In Appendix B, we show that $M$ and $N$ satisfy the relations

$$
\begin{aligned}
& M M^{*}-N N=I, \\
& M N^{*}-N M=0,
\end{aligned}
$$

where $I$ is the identity matrix.

Analytical expressions for $M$ and $N$ do not exist. However, it is possible to calculate the output signal vector $A\left(\xi_{0}\right)$ for any specific initial condition $A\left(-\xi_{0}\right)$ by solving Eq. (15) numerically. Even though calculating $M$ and $N$ is not neces- sary to find the gain, we evaluate these matrices since this form of the solution is particularly useful in bringing out the phase dependence of the gain. To calculate the $n$th column of these matrices, we solve Eq. (15) for the initial condition

$$
A\left(-\xi_{0}\right)=\exp \left(i \phi_{s}\right) \mathbf{a}_{n},
$$

where $\mathbf{a}_{n}$ is a unit vector whose only nonzero element is the $n$th element. The output vector corresponding to this initial condition is

$$
A\left(\xi_{0}\right)=\exp \left(i \phi_{s}\right) \sum_{m=0}^{\infty} \mathbf{a}_{m}\left[M_{m n}-i \exp (i \theta) N_{m n}\right] .
$$

To obtain the $n$th column of $M$ and $N$, we solve the output vector for two different initial condition phases, and use Eq. (17).

In our analysis, we use central finite differencing in the $\xi$ direction to solve Eq. (15). There are two sources of numerical error in these calculations. The first one is due to the finite step size $\Delta \xi$, where the error $\epsilon$ obeys the inequality

$$
\epsilon<(\gamma \Delta \xi)^{3} \frac{2 \xi_{0}}{\Delta \xi}
$$

In our calculations, we choose the step size in such a way that the error in the field amplitude is always less than $5 \times 10^{-4}$. The second source of error arises from truncating the infinite sum of Eq. (11) at a finite mode index $m=m_{s}$. However, the structure of $T_{m n}$ allows us to represent the signal field accurately with only the lower-order $m_{s}$ modes, when the input signal is composed of only a few lower-order modes. Under these circumstances, the power that couples to the higher-order modes $\left(m>m_{s}\right)$ is negligible. We have calculated that when the input signal consists of only the fundamental mode, the first 20 modes at the output are sufficient to represent the output signal field with an error that is a few orders of magnitude less than that due to the finite step size. One method we have used to investigate the accuracy of our results is to check if $N_{00}$ comes out to be a purely real quantity, a condition that is a consequence of $N$ being a Hermitian matrix.

Once the elements of $M$ and $N$ are found, the gain of the DOPA can be calculated for any input signal field. For an arbitrary signal input represented by the vector $A\left(-\xi_{0}\right)$ $=\exp (i \phi)\left[\begin{array}{llll}p_{0} & p_{1} & p_{2} & \cdots\end{array}\right]^{T}$, where $p_{n}$ are the coefficients of modal expansion, the small-signal gain of the DOPA can be written as

$$
g(\theta)=\sum_{m=0}^{\infty}\left|\sum_{n=0}^{\infty} M_{m n} p_{n}+\exp [i(\theta-\pi / 2)] N_{m n} p_{n}^{*}\right|^{2} .
$$

Note that at a fixed value of $\xi_{0}=l / 2 z_{0}$, the gain depends on both $\theta$ and $\gamma$. In Sec. IV, we use Eq. (23) to calculate the gain for a Gaussian input signal, and examine the dependence of the gain on the parameters $\theta, \gamma$, and $l / z_{0}$.

\section{QUADRATURE SQUEEZING}

Squeezed states of the light field are most commonly generated using DOPA's $[2,5]$. In this section, we consider the 
generation of squeezed vacuum states with a Gaussian-beampumped DOPA, and the detection of these states using a LO beam of arbitrary profile. Our analysis shows that, the measured squeezing is equal to the classical deamplification experienced by an arbitrary input signal to the DOPA, if the conjugate of that input were to be used as the LO beam in the detection of squeezed vacuum generated by the same DOPA. In particular, the maximum squeezing measured by a Gaussian LO beam is equal to the maximum deamplification that can be achieved for a Gaussian input signal in a DOPA. Therefore, the classical analysis described in Sec. II can be directly used for calculating the squeezing measured at the output of a DOPA.

In the plane-wave theory of DOPA's, quantizing Eq. (3) leads to the transformation [9]

$$
\hat{b}=\mu \hat{a}-i \exp \left(i \phi_{p}\right) \nu \hat{a}^{\dagger}
$$

where $\mu$ and $\nu$ are real quantities given by Eqs. (4) and (5), and $\hat{a}$ and $\hat{b}$ are the annihilation operators associated with the input and output signal plane waves, respectively. When the input signal is in a vacuum state, the output signal is in a squeezed vacuum state. Homodyne detection provides a measurement of the variance (noise) in an arbitrary quadrature of the squeezed field [12]. (In homodyne detection, a coherent-state plane-wave LO of frequency $\omega$ is mixed with the squeezed field, and the photon number of the resulting field is measured.) A LO having a phase of $\phi$ measures the field quadrature

$$
\hat{a}_{\phi}=\frac{1}{2}\left[\hat{a} \exp (-i \phi)+\hat{a}^{\dagger} \exp (i \phi)\right] .
$$

The input vacuum state has a mean $\left\langle\hat{a}_{\phi}\right\rangle=0$ and a variance $\left\langle\Delta \hat{a}_{\phi}^{2}\right\rangle=\left\langle\hat{a}_{\phi}^{2}\right\rangle-\left\langle\hat{a}_{\phi}\right\rangle^{2}=1 / 4$. The measured quadrature of the output squeezed vacuum state is

$$
\hat{b}_{\phi}=\frac{1}{2} \exp (-i \phi)[\mu+i \exp (-i \theta) \nu] \hat{a}+\text { H.c. }
$$

where H.c. stands for the Hermitian conjugate of the first term. This quadrature has a variance of

$$
\left\langle\Delta \hat{b}_{\phi}^{2}\right\rangle=\frac{1}{4}|\mu+i \exp (-i \theta) \nu|^{2},
$$

which depends on the phase difference $\theta=\phi_{p}-2 \phi$ between the pump and the LO. The ratio of the output and input variances is defined as the squeezing parameter

$$
S(\theta)=\frac{\left\langle\Delta \hat{b}_{\phi}^{2}\right\rangle}{\left\langle\Delta \hat{a}_{\phi}^{2}\right\rangle}=|\mu+i \exp (-i \theta) \nu|^{2} .
$$

Maximum squeezing occurs at $\theta=-\pi / 2$ where $S(\theta)$ assumes its smallest value of $(\mu-\nu)^{2}$. In this case, the measured quantum noise of the squeezed state becomes lower than the quantum limit by a factor of $R=1 / \min \{S(\theta)\}$. In the plane-wave theory, the maximum classical deamplification of the DOPA has the same value as maximum squeezing $R$; i.e., $\max \{S(\theta)\} \min \{S(\theta)\}=1$.
When the DOPA is pumped by a Gaussian beam, the input to the DOPA can be considered to be the set of all Laguerre-Gaussian modes, each mode being in the vacuum state. The annihilation operators of the input modes are independent of each other, and hence $\left[\hat{a}_{m}, \hat{a}_{n}^{\dagger}\right]=\delta_{m n}$. Since all the input modes are in the vacuum state, we have $\left\langle\hat{a}_{n \phi}\right\rangle=0$ and $\left\langle\Delta \hat{a}_{n \phi}^{2}\right\rangle=1 / 4$ for all $n$, where $\hat{a}_{n \phi}=1 / 2\left[\hat{a}_{n} \exp (-i \phi)\right.$ + H.c. $]$ is the measured quadrature of the $n$th mode.

Quantizing Eq. (17) leads to

$$
\hat{b}=M \hat{a}-i \exp \left(i \phi_{p}\right) N \hat{a}^{\dagger}
$$

where $\hat{a}$ and $\hat{b}$ are the input and output annihilation operator vectors, respectively. Equation (29) shows that each output mode

$$
\hat{b}_{m}=\sum_{n=0}^{\infty} M_{m n} \hat{a}_{n}-i \exp \left(i \phi_{p}\right) N_{m n} \hat{a}_{n}^{\dagger}
$$

is a weighted sum over all the input modes. The total output signal is

$$
\hat{b}_{s}(r)=\sum_{m=0}^{\infty} \hat{b}_{m} G_{m}\left(r, \xi_{d}\right)
$$

at the plane of detection $\xi=\xi_{d}$.

An arbitrary LO beam can be expressed as a sum of Laguerre-Gaussian modes. When all modes of the LO are in coherent states, the total LO field can be written as

$$
\hat{q}(r)=\sum_{m=0}^{\infty} q_{m} G_{m}\left(r, \xi_{d}\right) \hat{d}
$$

where $q_{m}$ are the complex constants of the mode expansion, and $\hat{d}$ is the annihilation operator corresponding to the coherent state LO.

In balanced homodyne detection, the LO field $\hat{q}$ is mixed with the squeezed field $\hat{b}_{s}$, and measurements of the photon numbers of the resulting beams are realized by two detectors whose photocurrents are subsequently subtracted [12]. The difference photocurrent operator is given by

$$
\hat{I}=2 \pi \int_{0}^{\infty}\left(\hat{b}_{s}^{\dagger} \hat{q}+\text { H.c. }\right) r d r .
$$

The orthonormality of the Laguerre-Gaussian modes reduces Eq. (33) to

$$
\hat{I}=\pi \sum_{m=0}^{\infty} q_{m} \hat{b}_{m}^{\dagger} \hat{d}+\text { H.c. }
$$

Substituting Eq. (30) into Eq. (34), interchanging the order of the summations, and using the properties that $M$ is symmetric and $N$ is Hermitian (see Appendix A), we obtain

$$
\hat{I}=\pi \sum_{n=0}^{\infty} \sum_{m=0}^{\infty} q_{m}\left[M_{n m}^{*} \hat{a}_{n}^{\dagger}+i \exp \left(-i \phi_{p}\right) N_{n m} \hat{a}_{n}\right] \hat{d}+\text { H.c. }
$$


When $\hat{d}$ is in a coherent state $|\alpha\rangle$ with mean value $\langle\alpha|\hat{d}| \alpha\rangle=|\alpha| \exp (i \phi)$, the variance of the difference photocurrent is

$$
\left\langle\Delta \hat{I}^{2}\right\rangle=\pi^{2} \sum_{n=0}^{\infty}\left|\sum_{m=0}^{\infty} M_{n m} q_{m}^{*}+i \exp (-i \theta) N_{n m} q_{m}\right|^{2}|\alpha|^{2},
$$

provided $|\alpha|^{2} \gg 1$. Since a vacuum state input yields $\left\langle\Delta \hat{I}^{2}\right\rangle$ $=\pi^{2}|\alpha|^{2}$, the squeezing parameter is

$$
S(\theta)=\sum_{m=0}^{\infty}\left|\sum_{n=0}^{\infty} M_{m n} q_{n}^{*}+\exp [-i(\theta-\pi / 2)] N_{m n} q_{n}\right|^{2},
$$

where the dummy indices $m$ and $n$ have been interchanged.

Comparing Eq. (37) with Eq. (23), we note that $g(\theta)$ and $S(\theta)$ are given by identical expressions if $q_{n}=p_{n}^{*}$, and the sign of the phase term $(\theta-\pi / 2)$ is reversed. We conclude that, except for a shift in phase, the dependence of the squeezing parameter on the phase difference between the pump and the LO is identical to the dependence of the classical gain of the DOPA on the phase difference between the pump and the signal input, provided the LO has the conjugate profile of the signal input. In the calculation of the classical gain we consider the coupling of a given set of modes at the input to all of the modes at the output, whereas for squeezing we consider the coupling of all of the input modes (each in the vacuum state) to a set of given output modes (determined by the LO beam). The equivalence between the classical and quantum solutions is a consequence of having the pump focus at the center of the crystal. The classical input and the LO beams are conjugates of each other since they are expressed on different sides of the pump focus. In Sec. IV, we use this equivalence to calculate the squeezing parameter for a Gaussian LO, and examine the dependence of squeezing on the parameters $\theta, \gamma$, and $l / z_{0}$.

\section{GAUSSIAN LOCAL OSCILLATOR}

In a typical squeezing experiment, the LO used for homodyne detection is a Gaussian beam that has the same confocal parameter as the pump. In other words, the LO beam

$$
\hat{q}(r)=G_{0}\left(r, \xi_{d}\right) \hat{d}
$$

is identical to the fundamental Laguerre-Gaussian beam used in the expansion of the signal field. In this section, we consider a Gaussian LO beam, and investigate the dependence of the measured squeezing level on various experimental parameters. In particular, we determine the limits imposed by GID on the maximum squeezing that can be measured.

The Gaussian LO of Eq. (38) measures only the $\hat{b}_{0}$ mode at the DOPA output, and the general expression of Eq. (37) for the squeezing parameter reduces to

$$
S(\theta)=\sum_{m=0}^{\infty}\left|M_{m 0}+\exp [-i(\theta-\pi / 2)] N_{m 0}\right|^{2} .
$$

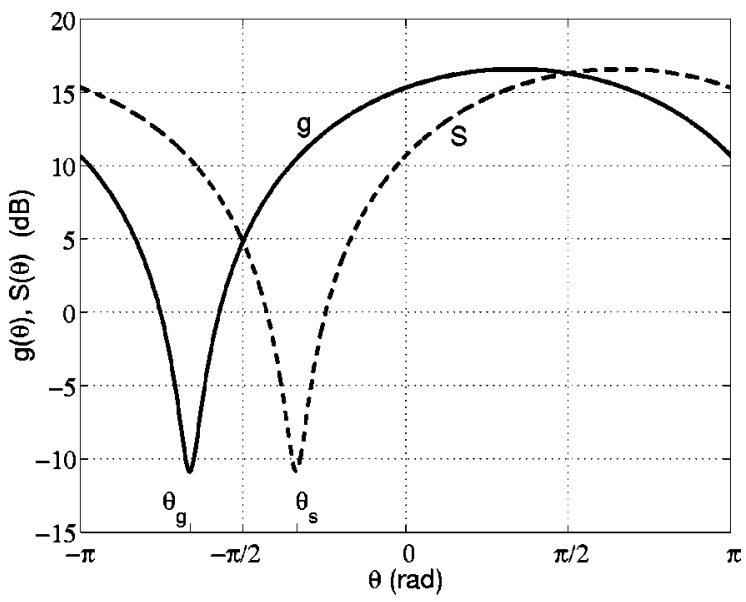

FIG. 2. DOPA gain $g(\theta)$ (solid line) and squeezing parameter $S(\theta)$ (dashed line) as functions of $\theta$, where $\gamma=4$ and $l / z_{0}=1$.

Equation (39) shows that there are contributions from all of the input modes in a measurement of the output fundamental mode. This effect is a direct consequence of GID.

As shown in Sec. III, the squeezing setup analyzed here is formally equivalent to the analysis of the gain of a classical DOPA with an input signal given by

$$
A\left(-\xi_{0}\right)=\exp \left(i \phi_{s}\right)\left[\begin{array}{llll}
1 & 0 & 0 & \cdots
\end{array}\right]^{T} .
$$

In this case, the classical small-signal gain expression of Eq. (23) reduces to

$$
g(\theta)=\sum_{m=0}^{\infty}\left|M_{m 0}+\exp [i(\theta-\pi / 2)] N_{m 0}\right|^{2} .
$$

In general, the value of $S$ depends on the parameters $\theta$, $l / z_{0}$, and $\gamma$. The constant

$$
\gamma=\kappa z_{0}\left|A_{p 0}\right|=\frac{2 d_{e}}{n c^{2}} \sqrt{\frac{2 \omega^{3} z_{0} P_{p}}{\pi \epsilon_{0}}}
$$

being proportional to the square root of pump power $P_{p}$ $=\pi z_{0} c^{2} \epsilon_{0}\left|A_{p 0}\right|^{2} / 8 \omega$ and the effective nonlinear coefficient $d_{e}$, is a measure of how strongly the DOPA is pumped. From an experimental point of view, however, the nonlinear drive defined as

$$
D=\left(\frac{8 \omega}{\pi c^{2} \epsilon_{0}}\right)\left(\kappa^{2} l P_{p}\right)=\left(\frac{l}{z_{0}}\right) \gamma^{2}
$$

is a more relevant parameter to investigate, since it is independent of the confocal parameter $z_{0}$. In a typical experimental setting, the maximum available $D$ is limited by the laser power, the crystal length, and the effective nonlinear coefficient. On the other hand, it is usually possible to adjust the value of $z_{0}$ by changing the focused spot size of the pump beam.

Figure 2 illustrates the phase dependences of the squeezing parameter and the classical gain through an example where $S(\theta)$ and $g(\theta)$ are plotted as functions of the phase difference $\theta$ for $l / z_{0}=1$ and $\gamma=4$. The minimum values of $S$ and $g$ occur at $\theta_{s}$ and $\theta_{g}$, respectively, both points being 

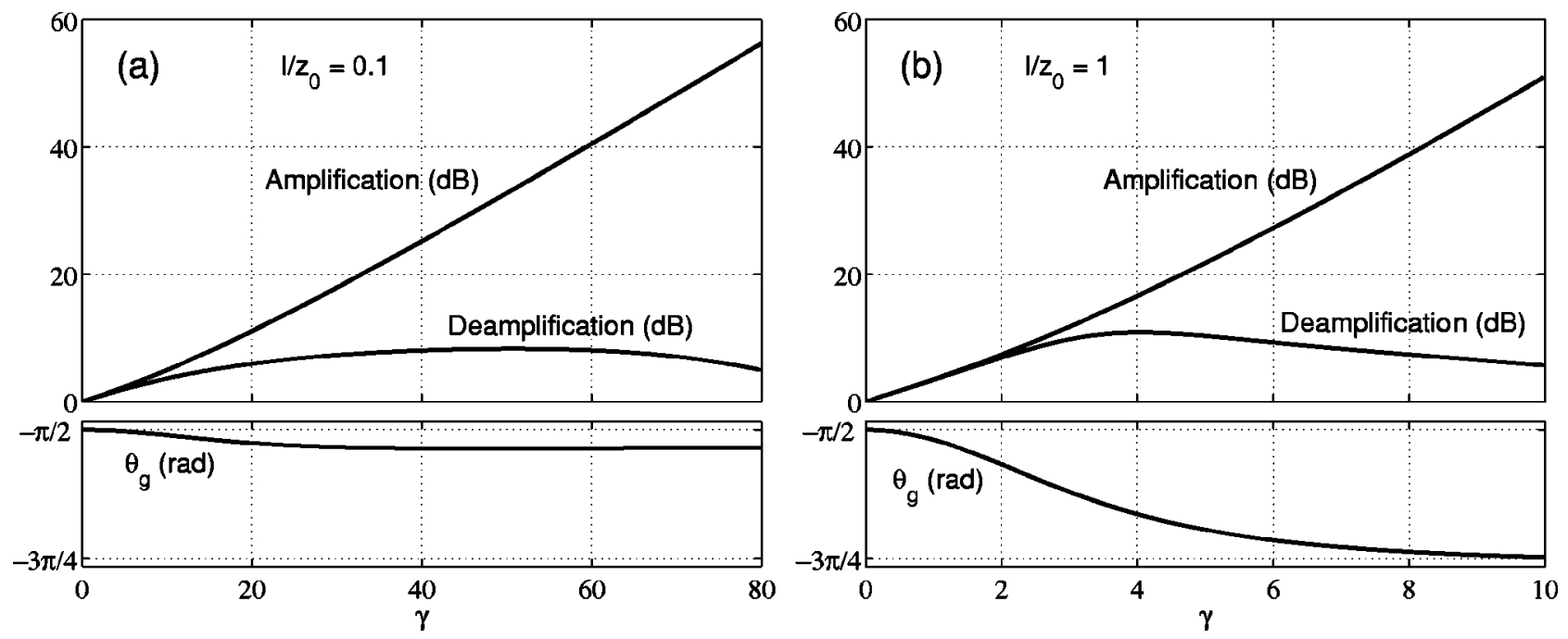

FIG. 3. Maximum (amplification) and minimum (deamplification) values of DOPA gain $g(\theta)$, and the optimum phase difference $\theta_{g}$ as functions of $\gamma$; (a) $l / z_{0}=0.1$ and (b) $l / z_{0}=1$.

markedly different from $-\pi / 2$, the value predicted by the plane-wave theories. Note that a false assumption that maximum squeezing (minimum $S$ ) occurs at $\theta=-\pi / 2$ results in an error that is greater than $15 \mathrm{~dB}$. On the other hand, the phase separation between the maximum and minimum points of $S$ is $\pi$, just as in the plane-wave theory. Furthermore, a comparison of Eqs. (23) and (37) shows that $\theta_{s}+\theta_{g}=-\pi$. This relation, and the symmetry property of $g$ and $S$ with respect to $\theta=-\pi / 2$, are independent of the value of $l / z_{0}$. This is a consequence of defining $\theta$ at the center of the crystal. For the alternative definition at the input plane, one has $\theta_{s}^{\prime}+\theta_{g}^{\prime}=-\pi+2 \tan ^{-1}\left(l / z_{0}\right)$ instead.

Another important quantity is the sensitivity of $S(\theta)$ to the fluctuations in the phase difference $\theta$ about its minimum point $\theta_{s}$, in other words, the sharpness of the dip in Fig. 2. In an experiment, the phase difference between the pump and the LO beams fluctuates in time by a certain amount, even when active stabilization is employed. These fluctuations may wash out the squeezing level during the measurement [8]. We define the normalized $10 \%(0.41 \mathrm{~dB})$ phase width of the squeezing parameter as $\delta=\Delta \theta / 2 \pi$, where $\Delta \theta$ is the full width at $1.1 \min \{S\}$. (For the example shown in Fig. 2, $\delta$ $=8.3 \times 10^{-3}$.) In an experiment, if the phase fluctuations become larger than $\delta$, the maximum squeezing that can be detected begins to deviate from $1 / \min \{S\}$ significantly. Therefore, it is desirable to operate at a large $\delta$ value.

Figure 3 shows the maximum classical amplification and deamplification [maximum and minimum values of $g(\theta)$ ] as functions of $\gamma$ at $l / z_{0}=0.1$ and $l / z_{0}=1$. The $\theta_{g}$ values at which the deamplification is maximized are also included in the figure for each $\gamma$ value. Note that, in the limit as $\gamma$ approaches zero, $\theta_{g}$ approaches the plane-wave theoretic value of $-\pi / 2$, regardless of the value of $l / z_{0}$. This is expected since in this regime GID effects disappear. (The limit becomes dependent on $l / z_{0}$ if the phase difference is defined at the input plane.)

As $\gamma$ is increased, the amplification of the DOPA increases without bound. However, maximum deamplification is achieved at a particular value of $\gamma$, above which the deamplification decreases. This behavior is a consequence of GID; the phase fronts of the signal beam are distorted as the beam propagates through the DOPA crystal. Here the fundamental mode itself may be deamplified more than the overall beam; however, the coupling of the fundamental mode to the higher-order modes results in an overall decrease in the deamplification. Also note that, the product of maximum amplification and deamplification is close to unity (as in the plane-wave theory) for small values of $\gamma$, where GID effects are less pronounced. However, for large values of $\gamma$ this is no longer true, and GID effects may become so large that $g(\theta)$ may be greater than unity for all values of $\theta$ (for $\gamma$ $>15$ in the $l / z_{0}=1$ example). Figure 4 illustrates the phasefront distortions due to GID, where the phase fronts of the signal and the pump beams are shown for $\gamma=4, l / z_{0}=1$, and $\theta=\theta_{g}$. Note the increased divergence of the signal beam towards the output.

At a fixed value of $l / z_{0}$, the squeezing parameter $S$ depends on both $\theta$ and $\gamma$. In our analysis, we compute the $\theta$

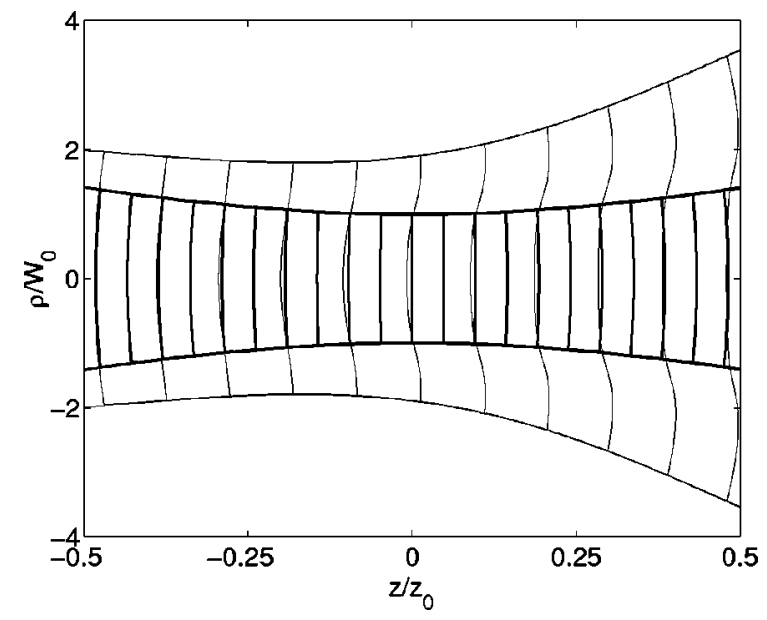

FIG. 4. Phase fronts of the pump and signal beams inside the nonlinear crystal illustrating distortions due to GID, where $\gamma=4$, $l / z_{0}=1$, and $\theta=\theta_{g}$. The bounding curves show the beam radii (1/e $e^{2}$ of peak intensity). For clarity, the wavelength is exaggerated and the pump phase is shifted by $\pi / 2$. 

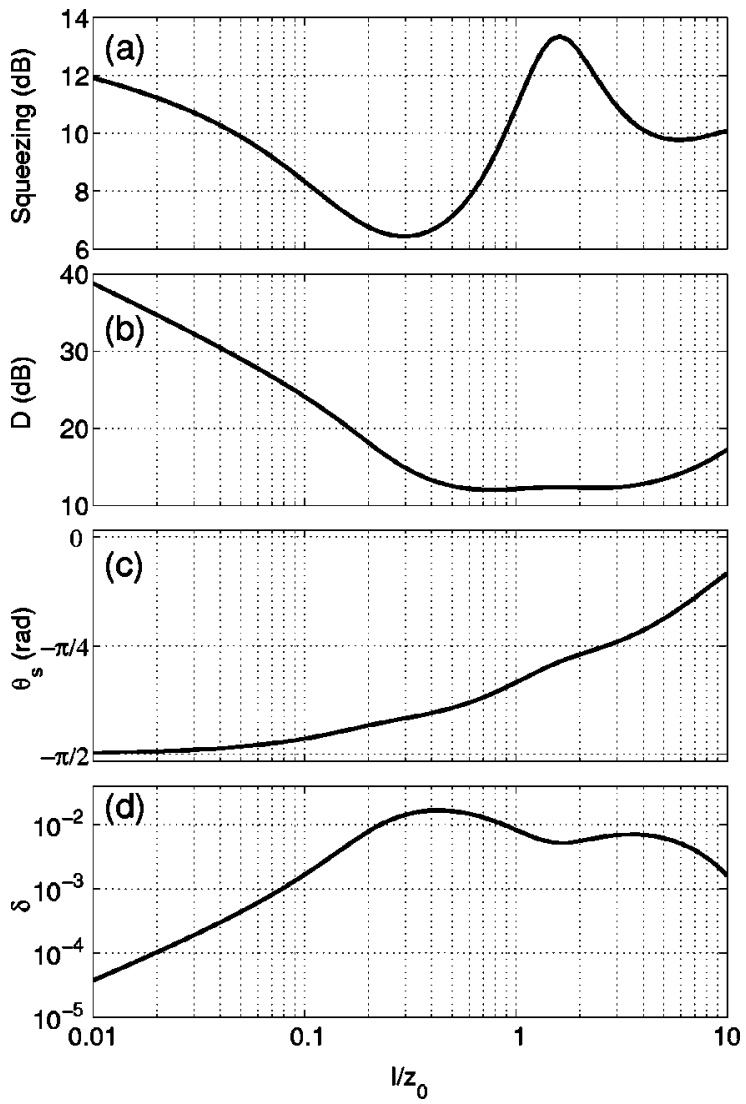

FIG. 5. (a) Maximum squeezing, (b) required nonlinear drive $D$, (c) optimum phase difference $\theta_{s}$, and (d) normalized $10 \%(0.41$ $\mathrm{dB})$ phase width $\delta$ as functions of $l / z_{0}$.

and $\gamma$ values that minimize the squeezing parameter by using numerical optimization algorithms. Figure 5 shows the maximum possible squeezing (deamplification) as a function of $l / z_{0}$. The $\theta$ and $D$ values that maximize squeezing at each $l / z_{0}$ value are also included in the figure. Note that for each $l / z_{0}$ value, $D$ and $\gamma$ are related through Eq. (43). The normalized phase width $\delta$ is also included in the figure.

For small values of $l / z_{0}$, the phase difference that maximizes the squeezing level $\theta_{s}$ is close to $-\pi / 2$; this is as expected since GID effects are small in this regime. Squeezing levels larger than $10 \mathrm{~dB}$ can be realized for $l / z_{0}<0.04$. However, in this regime the nonlinear drive $D$, and hence the pump power, required to obtain maximum squeezing are very high. Also note that, the normalized phase width $\delta$ is very narrow, less than $3 \times 10^{-4}$ for $l / z_{0}<0.04$.

Squeezing decreases with increasing $l / z_{0}$ up to $l / z_{0}$ $=0.3$, while $\theta_{s}$ deviates from $-\pi / 2$, the required $D$ decreases, and $\delta$ increases. However for $l / z_{0}>0.3$, squeezing begins to increase while the required $D$ levels off at about 12 $\mathrm{dB}$. Even though $\delta$ begins to decrease from its maximum value at $l / z_{0}=0.3$, it nevertheless stays relatively high. Squeezing levels in excess of $10 \mathrm{~dB}$ can be obtained with relatively low nonlinear drive in the $0.9<l / z_{0}<4.2$ range. In this regime, $\theta_{s}$ is significantly different from $-\pi / 2$, and $\delta$ stays greater than $\sim 5 \times 10^{-3}$. The highest squeezing is 13.4 $\mathrm{dB}$ and occurs at $l / z_{0}=1.6$, where the optimum $D$ is 12.3 $\mathrm{dB}$, and $\delta=5.2 \times 10^{-3}$.

Even though squeezing in excess of $10 \mathrm{~dB}$ is possible for $l / z_{0}<0.04$, the required nonlinear drive is likely to prohibit working in this regime. The nonlinear drive required to achieve $11.9 \mathrm{~dB}$ squeezing at $l / z_{0}=0.01$ is approximately 450 times that at $l / z_{0}=1.15$ (or 2.35) for the same squeezing level. Furthermore, operating at $l / z_{0}=0.01$ requires a phase stability that is 160 times better than that at $l / z_{0}=1.15$ (or 2.35). The advantages of working in the large $l / z_{0}$ regime are clear.

A comparison of our formulation with previous models is provided in Fig. 6. For two different $l / z_{0}$ values, we plot squeezing (deamplification) as a function of $\gamma$ using $(A)$ the model of Ref. [8], $(B)$ the model of Ref. [6], $(C)$ our model with $\theta=-\pi / 2$, and $(D)$ our model with $\theta=\theta_{s}$. For the $l / z_{0}=0.1$ case in Fig. 6(a), the results of Ref. [8] (A) are essentially reproduced by our model when we set $\theta=$ $-\pi / 2(C)$, both predicting about the same deamplification at the same $\gamma$. The model of Ref. [6] (B), on the other hand, overestimates the squeezing level since the Gouy phase of the pump is neglected in these calculations. When we set $\theta$ to its optimum value $\theta_{s}$ in our model $(D)$, we find higher levels of squeezing. On the other hand for the $l / z_{0}=1$ case in Fig. 6(b), the formulation of Ref. [8] (A) is no longer appli-

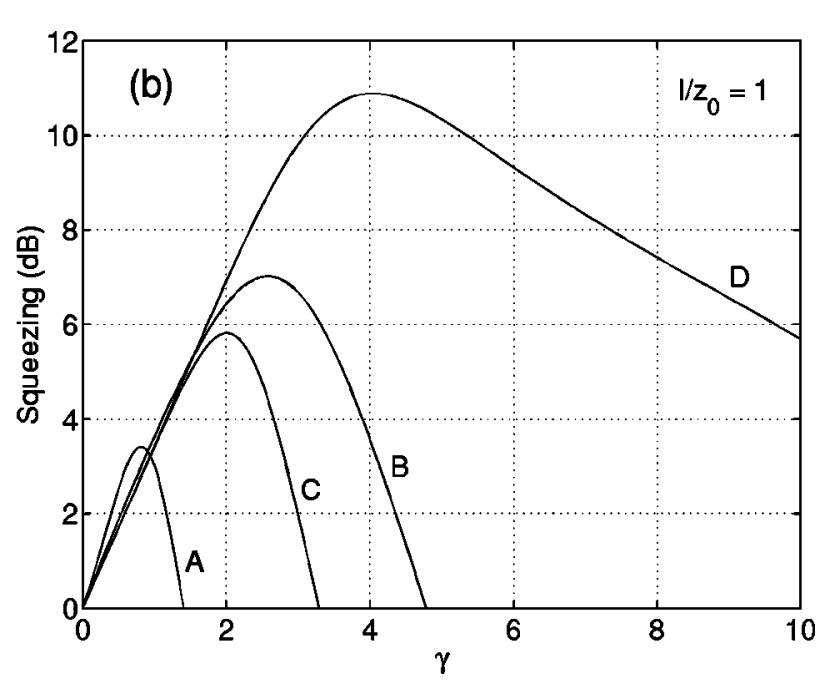

FIG. 6. Deamplification (or squeezing) a function of $\gamma$ calculated using $(A)$ the model of Ref. [8], $(B)$ the model of Ref. [6], $(C)$ our model with $\theta=-\pi / 2$, and $(D)$ our model with $\theta=\theta_{s}$; (a) $l / z_{0}=0.1$ and (b) $l / z_{0}=1$. 
cable, since this model is valid only for $l / z_{0} \ll 1$. The model of Ref. [6] (B) would have been applicable in this large $l / z_{0}$ regime, had it contained the necessary Gouy phase and optimized the phase difference $\theta$. We conclude that the squeezing limits calculated in Ref. [6] are not valid. On the other hand, the model of Ref. [8] is not applicable in the high- $l / z_{0}$ regime where large levels of squeezing at low nonlinear drive values are possible.

The quantum efficiency of homodyne detection plays an important role in squeezing experiments. For a quantum efficiency of $\eta$, the relation between the generated squeezing parameter $S$ and the measured squeezing parameter $S_{m}$ is given by [12]

$$
S_{m}=\eta S+(1-\eta)
$$

Therefore, measured squeezing is limited to $1 /(1-\eta)$, regardless of the value of $S$.

The conditions for validity and the limitations of the DOPA model described in this paper should be carefully pointed out. The perfect phase-matching condition assumed at the beginning breaks down when $l / z_{0}$ is so large that the beam divergence angle becomes comparable to or greater than the crystal acceptance angle. This is why we have limited our investigation to $l / z_{0} \leqslant 10$. (The acceptance angle of typical nonlinear crystals can be as large as a few degrees; an $l / z_{0}$ value of 10 implies a pump beam divergence less than $2^{\circ}$ at a pump wavelength of $500 \mathrm{~nm}$ and $l=10 \mathrm{~mm}$.) On the other hand, our model assumes that there is no walk-off in the transverse plane between the pump and the signal beams. This condition is satisfied if noncritical phase matching is employed. However, for critically phase-matched DOPA's our model may not be accurate, depending on the magnitude of the walk-off angle. Furthermore, our model is only approximately valid for type-II phase-matched DOPA's. A more accurate analysis should consider the signal and idler modes separately during the interaction, and combine them at the output. Modeling of pulsed DOPA's should take the temporal profiles of the pulses into account.

The results shown in Fig. 5 do not reflect the absolute limits of squeezing even within the confines of our model. In the beginning of our analysis, we assumed that the confocal parameters of the signal and pump beams are equal. However, we have found that by allowing the signal and the pump to have different $z_{0}$ values, it is possible to increase the squeezing level, as previously noted in Ref. [7]. For example at $l / z_{0}=1.6$ where squeezing is maximum in Fig. 5, having a 0.91 ratio between the confocal parameters of the signal and the pump beams improves squeezing by $0.7 \mathrm{~dB}$. Furthermore, placing the pump focus at the center of the crystal may not be the optimum strategy either [7].

In summary, to achieve large levels of squeezing in an experiment where the LO is a Gaussian beam, it is necessary to choose $l / z_{0} \approx 1.6$ and adjust the pump power to realize the corresponding optimum $D$ value (approximately $12 \mathrm{~dB}$ ). This strategy requires much less pump power and phase stability compared to working in the weak focusing ( regime. A squeezing experiment using a Gaussian LO is capable of achieving about $13 \mathrm{~dB}$ of squeezing, provided there is adequate phase stability and high quantum efficiency.

\section{MATCHED LOCAL OSCILLATOR}

The main reason for using a Gaussian $\mathrm{LO}$ beam to detect squeezing is experimental convenience. As a consequence of GID, however, a Gaussian LO is not matched to extract the maximum squeezing at the output of a Gaussian-beam pumped DOPA. A LO that is perfectly matched to the DOPA output would be a beam whose mode expansion coefficients $q_{n}$ minimize $S$ as given in Eq. (37). There may also be LO beams that are matched better than a Gaussian but worse than a perfectly matched LO.

The use of matched LO beams for the detection of quadrature squeezing was reported in two experiments $[10,11]$. Both are based on generating a matched LO beam with an OPA that is identical or similar to the one used for generating squeezed vacuum. The idea is to mimic the phase and amplitude distortions of the squeezed field on the LO beam so that the two beam profiles may match each other.

In this section, we investigate the generation of a matched LO through the amplification of a Gaussian beam with a DOPA that is identical to the one used for generating a squeezed vacuum. We show that the squeezing measured with such a matched LO is equal to the amplification experienced by the Gaussian beam input. In contrast to deamplification, amplification with a DOPA is not bounded for a Gaussian input, as shown in Fig. 3 and discussed in Sec. IV. Therefore, the use of a matched LO beam alleviates the GIDimposed limits on squeezing.

We consider two identical DOPA's; the first one is used for amplifying an input Gaussian beam, and the second one for generating squeezed vacuum. The output of the first DOPA is then used to measure the squeezed vacuum at the output of the second DOPA in a balanced homodyne configuration. Later in this section, we discuss that the scheme analyzed here is identical to the self-generated matched LO experiment reported in Ref. [11], and approximately valid for the nondegenerate OPA (NOPA) experiment of Ref. [10].

The Gaussian input to the first DOPA is represented by the initial condition given in Eq. (40). Using Eq. (17), the output field can be written as

$$
A\left(\xi_{0}\right)=M \mathbf{a}_{0}+\exp \left[i\left(\theta_{1}-\pi / 2\right)\right] N \mathbf{a}_{0},
$$

where the unit vector $\mathbf{a}_{0}=\left[\begin{array}{llll}1 & 0 & 0 & \cdots\end{array}\right]^{T}$, and $\theta_{1}=\phi_{p 1}$ $-2 \phi_{s}$ is the phase difference between the pump and the input signal of this DOPA. The gain is given by Eq. (41), and equal to $g\left(\theta_{1}\right)=\left\|A\left(\xi_{0}\right)\right\|^{2}$, since the input signal has unity amplitude. Note that $g\left(\theta_{1}\right)$ assumes its maximum value at $\theta_{1}=\theta_{g}+\pi$.

The output beam given in Eq. (45) is used as the LO to detect squeezing at the output of the second DOPA. The mode expansion coefficients of the LO beam are the elements of the vector

$$
q=\frac{A\left(\xi_{0}\right)}{\left\|A\left(\xi_{0}\right)\right\|} .
$$

Note that, even though the quantum state of this LO beam is not a coherent state, Eq. (37) is still valid since any excess LO noise is canceled in the balanced homodyne configuration $[11,13]$. Using Eq. (37), the squeezing detected by the matched $\mathrm{LO}$ beam can be written as 


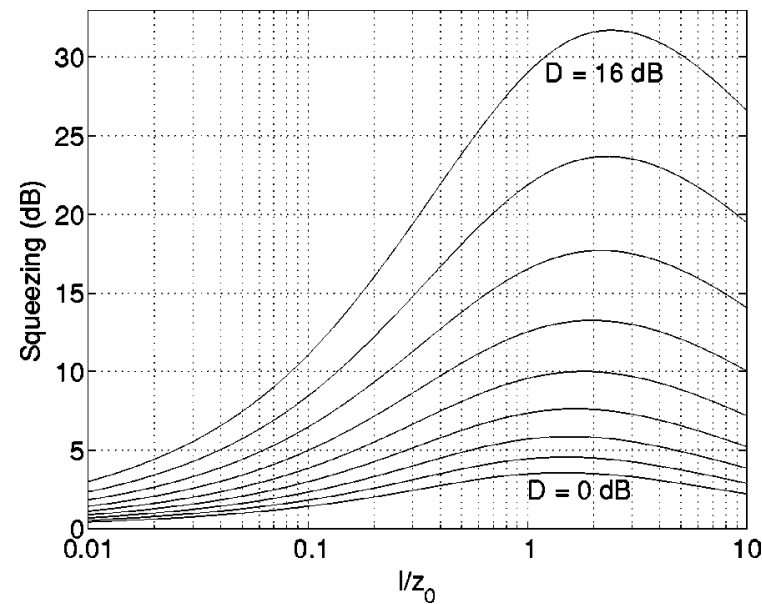

FIG. 7. Squeezing measured with a matched local oscillator as a function of $l / z_{0}$ at constant nonlinear drive values $D$ ranging from 0 to $16 \mathrm{~dB}$ in $2-\mathrm{dB}$ increments.

$$
S\left(\theta_{1}, \theta_{2}\right)=\left\|M q^{*}+\exp \left[-i\left(\theta_{2}-\pi / 2\right)\right] N q\right\|^{2},
$$

where $\theta_{2}=\phi_{p 2}-2 \phi$ is the phase difference between the pump of the second DOPA and the LO. Note that, the squeezing parameter depends on both $\theta_{1}$ and $\theta_{2}$.

Substituting Eq. (46) into Eq. (47) and using the properties $M N^{*}-N M=0$ and $M M^{*}-N N=I$ (see Appendix B), the squeezing parameter becomes

$$
\begin{aligned}
S\left(\theta_{1}, \theta_{2}\right)= & \frac{1}{\left\|A\left(\xi_{0}\right)\right\|^{2}} \|\left\{1+\exp \left[-i\left(\theta_{2}-\theta_{1}\right)\right]\right\} M M^{*} \mathbf{a}_{0} \\
& +\left\{\exp \left[-i\left(\theta_{1}-\pi / 2\right)\right]\right. \\
& \left.+\exp \left[-i\left(\theta_{2}-\pi / 2\right)\right]\right\} M N^{*} \mathbf{a}_{0} \\
& -\exp \left[-i\left(\theta_{2}-\theta_{1}\right)\right] I \mathbf{a}_{0} \|^{2} .
\end{aligned}
$$

Regardless of the value of $\theta_{1}$, the LO phase $\phi$, and hence $\theta_{2}$, can be adjusted so that $\theta_{2}-\theta_{1}=\pi$, at which point Eq. (48) reduces to

$$
S\left(\theta_{1}\right)=\frac{1}{\left\|A\left(\xi_{0}\right)\right\|^{2}}=\frac{1}{g\left(\theta_{1}\right)} .
$$

Note that, Eq. (49) is the minimum of Eq. (48) when $\theta_{1}$ is held constant and $\theta_{2}$ is varied. Equation (49) shows that the measured squeezing level is identical to the classical gain.

Equation (49) assumes its lowest value when the gain of the first DOPA is maximized by adjusting $\phi_{s}$ so that $\theta_{1}$ $=\theta_{g}+\pi$. We conclude that the highest squeezing level measured by the matched LO is equal to the maximum amplification experienced by the Gaussian beam input, i.e.,

$$
R=\max \left\{g\left(\theta_{1}\right)\right\} .
$$

The maximum amplification that can be achieved with a DOPA is not bounded for a Gaussian input beam (see Fig. 3 ). Therefore, squeezing is limited only by the available nonlinear drive $D$.

Figure 7 shows the maximum squeezing level as a function of $l / z_{0}$ at a number of fixed $D$ values, where $\theta_{1}$ and $\theta_{2}$
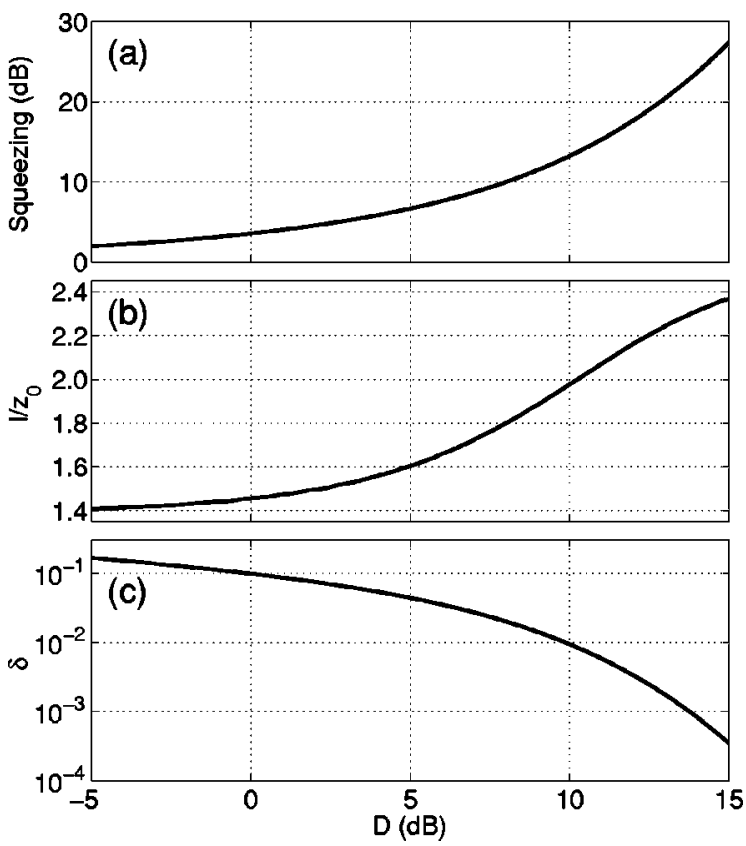

FIG. 8. (a) Maximum squeezing, (b) optimum $l / z_{0}$, and (c) normalized phase width $\delta$ as functions of available nonlinear drive $D$.

have both been optimized at each $D$ and $l / z_{0}$ point. For a fixed nonlinear drive $D$, squeezing increases rapidly with increasing $l / z_{0}$, reaching a maximum at some relatively large value of $l / z_{0}$. The peak of each curve in Fig. 7 represents the maximum possible squeezing that can be achieved at that nonlinear drive value. Figure 8 shows the maximum possible squeezing and the optimal $l / z_{0}$ value as functions of the available nonlinear drive $D$. The normalized phase width $\delta$ is also given at each nonlinear drive. For the range of $D$ values covered in Fig. 8, the optimum $l / z_{0}$ is in the 1.4-2.4 range. Therefore, we conclude that, just as in the case of a Gaussian LO, operating in the large $l / z_{0}$ regime is desirable when using a matched LO as well.

The advantages of detection with a matched LO rather than a Gaussian LO are clear. In the matched LO case, the squeezing that can be measured is only limited by the available nonlinear drive $D$, whereas in the case of a Gaussian LO it is limited to about $13 \mathrm{~dB}$ because of GID. A comparison of Figs. 8 and 5 provides some quantitative insight into the advantages of using a matched LO. With a Gaussian LO, a nonlinear drive of $12 \mathrm{~dB}$ is needed for generating about 13- $\mathrm{dB}$ squeezing. The use of a matched LO, on the other hand, yields a squeezing level of $18.4 \mathrm{~dB}$ at the same nonlinear drive. On the other hand, the quantum efficiency of homodyne detection $\eta$ may be the real limiting factor in an experimental situation. In such a case, using a matched LO (or any other method that increases squeezing) would not improve the measured squeezing level much. For example, if $\eta=0.8$, the $5-\mathrm{dB}$ increase in squeezing gained by using a matched LO shrinks to a mere $0.5 \mathrm{~dB}$ in measured squeezing.

The analysis presented in this section applies directly to the self-generated matched LO experiment reported in Ref. [11]. In this experiment, a type-II phase-matched OPA is used for generating squeezed vacuum. The signal mode of the squeezing DOPA is polarized at a $\sim 45^{\circ}$ angle to the signal and idler modes of the type-II OPA. However, the 
nondegeneracy in polarization allows this OPA to be used as two identical DOPA's that have orthogonally polarized signal modes [11]. A Gaussian input to the orthogonally polarized DOPA mode facilitates the generation of a matched LO by the same OPA. Note that the self-generated LO scheme cannot be used with type-I DOPA's. However, an identical but separate DOPA can still be used for generating a matched LO beam.

In the experiment reported in Ref. [10], squeezing is generated with a type-II OPA similar to the one in Ref. [11]. However, in this experiment the idler output of a NOPA is used as the matched LO beam. Analyzing this configuration, we have found that this LO beam is matched to the squeezed field much better than a Gaussian LO, although not as good as the self-generated LO. However, the difference between the two matched LO's is minimal when the classical gain of the LO generating OPA is high. This is as expected, since the difference between the idler and signal fields decreases rapidly with increasing gain.

In summary, a matched LO beam can be generated using a DOPA that is identical to the one used for generating squeezed vacuum. The squeezing that can be generated and detected with such a setup is limited only by the available nonlinear drive, the phase stability, and the quantum efficiency.

\section{CONCLUSION}

In this paper, we have presented a detailed and accurate model of squeezed-state generation using Gaussian-beam pumped DOPA's. This model incorporates diffractive effects resulting from transverse gain variations in the tight focusing regime as well as the weak.

The theoretical framework of this paper has shown that for a Gaussian-beam pumped DOPA, there is a formal equivalence between the classical small-signal gain and the squeezing parameter, just as in the plane-wave theory. According to this, the classical deamplification experienced by an arbitrary input signal beam is equal to the measured squeezing, when the conjugate of this beam is used as the LO in the homodyne detection of squeezed vacuum generated by the same DOPA (except for a shift in phase).

Applying our formalism to the case of a Gaussian LO beam, we found that in general the phase difference that maximizes squeezing depends on the ratio of the crystal length to the confocal parameter of the pump beam $\left(l / z_{0}\right)$. Even though this phase difference is close to the plane-wave theoretic value of $-\pi / 2$ for weak focusing $\left(l / z_{0} \ll 1\right)$, it deviates from this value significantly as the focus becomes tighter. More significantly, the squeezing predicted at a phase difference of $-\pi / 2$ is usually several $\mathrm{dB}$ lower than that at the optimum phase difference. This behavior is not particular to the Gaussian LO case, but applies to any LO beam.

In the Gaussian LO case, GID effects impose a limit on the maximum squeezing that can be achieved. This limit depends on the value of $l / z_{0}$, and achieving it requires a specific pump power (nonlinear drive). In the weak focusing regime, even though the limit on squeezing is not stringent, the pump power required to achieve it is prohibitively high. In the tight focusing regime, choosing $l / z_{0} \approx 1.6$, about 13
$\mathrm{dB}$ of squeezing can be generated with much less pump power. Another advantage of operating in this regime is the reduced phase stability requirements in the experimental setup, as reflected in the much larger phase-width values $\delta$.

In this paper, we have also investigated the generation and use of matched LO beams in the detection of squeezed vacuum at the output of a Gaussian-beam pumped DOPA. A matched LO generated by amplifying a Gaussian input signal with a DOPA is found to alleviate GID-induced limits on squeezing. In this scheme, we found that the measured squeezing is equal to the classical amplification of the LOgenerating DOPA, and is limited only by the available pump power.

\section{ACKNOWLEDGMENTS}

The authors wish to thank Dr. E. Sezer and Dr. I. Aksun for valuable discussions.

\section{APPENDIX A: $M$ IS SYMMETRIC AND $N$ IS HERMITIAN}

In this appendix, we show that the matrices $M$ and $N$ that appear in Eq. (17) are, respectively, symmetric and Hermitian, provided that

$$
T^{\prime}=\frac{T}{-i \exp \left(i \phi_{p}\right)}
$$

satisfies the conditions

$$
\begin{gathered}
T^{\prime}(-\xi)=T^{\prime *}(\xi), \\
T^{\prime T}=T^{\prime},
\end{gathered}
$$

where $T$ is the coefficient matrix of Eq. (15). Since the beam waist of the pump is located at the center of the nonlinear crystal, these conditions are satisfied by the coefficient matrix whose elements are given in Eq. (16).

Consider the solution of

$$
\frac{d}{d \xi} A=T^{\prime} A^{*}
$$

where the elements of $A$ and $T^{\prime}$ are complex quantities. Equation (A4) can be converted to an equation involving only real quantities using

$$
\begin{aligned}
& A=X+i Y, \\
& T^{\prime}=U+i V
\end{aligned}
$$

to yield

$$
\frac{d}{d \xi}\left[\begin{array}{l}
X \\
Y
\end{array}\right]=\left[\begin{array}{cc}
U & V \\
V & -U
\end{array}\right]\left[\begin{array}{l}
X \\
Y
\end{array}\right]
$$

The linearity of Eq. (A7) allows us to write the solution at $\xi$, given the initial condition at $\xi^{\prime}\left(\xi>\xi^{\prime}\right)$, using a state transition matrix $\psi\left(\xi, \xi^{\prime}\right)$ as

$$
\left[\begin{array}{l}
X(\xi) \\
Y(\xi)
\end{array}\right]=\left[\begin{array}{ll}
\psi_{11}\left(\xi, \xi^{\prime}\right) & \psi_{12}\left(\xi, \xi^{\prime}\right) \\
\psi_{21}\left(\xi, \xi^{\prime}\right) & \psi_{22}\left(\xi, \xi^{\prime}\right)
\end{array}\right]\left[\begin{array}{l}
X\left(\xi^{\prime}\right) \\
Y\left(\xi^{\prime}\right)
\end{array}\right] .
$$


For the problem at hand, the initial condition is specified at $\xi=-\xi_{0}$ and the solution is sought at $\xi=\xi_{0}$. Therefore, a new state transition matrix can be defined as $\Psi\left(\xi_{0}\right)$ $=\psi\left(\xi_{0},-\xi_{0}\right)$. Taking the derivative of $\Psi\left(\xi_{0}\right)$ and using Eqs. (A2) and (A3), we obtain

$$
\begin{aligned}
\frac{d}{d \xi_{0}}\left[\begin{array}{cc}
\Psi_{11} & \Psi_{12} \\
\Psi_{21} & \Psi_{22}
\end{array}\right]= & {\left[\begin{array}{cc}
U & V \\
V & -U
\end{array}\right]\left[\begin{array}{cc}
\Psi_{11} & \Psi_{12} \\
\Psi_{21} & \Psi_{22}
\end{array}\right] } \\
& +\left[\begin{array}{cc}
\Psi_{11} & \Psi_{12} \\
\Psi_{21} & \Psi_{22}
\end{array}\right]\left[\begin{array}{cc}
U & -V \\
-V & -U
\end{array}\right] .
\end{aligned}
$$

We define a new matrix $\Gamma=\Psi-P \Psi^{T} P$, where

$$
P=\left[\begin{array}{cc}
I & 0 \\
0 & -I
\end{array}\right]
$$

and $I$ is the identity matrix. The matrix $\Gamma$ satisfies

$$
\begin{aligned}
\frac{d}{d \xi_{0}}\left[\begin{array}{ll}
\Gamma_{11} & \Gamma_{12} \\
\Gamma_{21} & \Gamma_{22}
\end{array}\right]= & {\left[\begin{array}{cc}
U & V \\
V & -U
\end{array}\right]\left[\begin{array}{ll}
\Gamma_{11} & \Gamma_{12} \\
\Gamma_{21} & \Gamma_{22}
\end{array}\right] } \\
& +\left[\begin{array}{ll}
\Gamma_{11} & \Gamma_{12} \\
\Gamma_{21} & \Gamma_{22}
\end{array}\right]\left[\begin{array}{cc}
U & -V \\
-V & -U
\end{array}\right] .
\end{aligned}
$$

Note that $\Psi(0)=I$ when $\xi_{0}=0$, which implies that $\Gamma(0)$ $=0$. With this initial condition, the solution of Eq. (A11) becomes $\Gamma\left(\xi_{0}\right)=0$ for all $\xi_{0}$. Using the defining equation for $\Gamma$, we can write

$$
\left[\begin{array}{ll}
\Psi_{11} & \Psi_{12} \\
\Psi_{21} & \Psi_{22}
\end{array}\right]=\left[\begin{array}{cc}
\Psi_{11}^{T} & -\Psi_{21}^{T} \\
-\Psi_{12}^{T} & \Psi_{22}^{T}
\end{array}\right]
$$

Therefore, the solution of Eq. (A4) can be rewritten as

$$
\left[\begin{array}{c}
X\left(\xi_{0}\right) \\
Y\left(\xi_{0}\right)
\end{array}\right]=\left[\begin{array}{cc}
\Psi_{11}\left(\xi_{0}\right) & \Psi_{12}\left(\xi_{0}\right) \\
-\Psi_{12}^{T}\left(\xi_{0}\right) & \Psi_{22}\left(\xi_{0}\right)
\end{array}\right]\left[\begin{array}{c}
X\left(-\xi_{0}\right) \\
Y\left(-\xi_{0}\right)
\end{array}\right]
$$

or, in complex form, as

$$
A\left(\xi_{0}\right)=M A\left(-\xi_{0}\right)+N A^{*}\left(-\xi_{0}\right),
$$

where the matrices $M$ and $N$ are given by

$$
\begin{aligned}
& M=\left(\frac{\Psi_{11}+\Psi_{22}}{2}-i \frac{\Psi_{12}+\Psi_{12}^{T}}{2}\right), \\
& N=\left(\frac{\Psi_{11}-\Psi_{22}}{2}+i \frac{\Psi_{12}-\Psi_{12}^{T}}{2}\right) .
\end{aligned}
$$

As a consequence of Eq. (A12), the matrices $M$ and $N$ are found to be symmetric and Hermitian, respectively.

The solution of Eq. (15) can be obtained using the solution of Eq. (A4). Rewriting Eq. (15) as

$$
\frac{d}{d \xi} A=-i \exp \left(i \phi_{p}\right) T^{\prime} A^{*}
$$

and using the transformation $A^{\prime}=A \exp \left(\phi_{p} / 2-\pi / 4\right)$, Eq. (A17) is converted to

$$
\frac{d}{d \xi} A^{\prime}=T^{\prime} A^{\prime *}
$$

From Eq. (A14), the solution of $A^{\prime}$ is found to be

$$
A^{\prime}\left(\xi_{0}\right)=M A^{\prime}\left(-\xi_{0}\right)+N A^{\prime} *\left(-\xi_{0}\right),
$$

from which we can write the solution of $A$ as

$$
A\left(\xi_{0}\right)=M A\left(-\xi_{0}\right)-i \exp \left(i \phi_{p}\right) N A^{*}\left(-\xi_{0}\right)
$$

\section{APPENDIX B: $M M^{*}-N N=I$ AND $M N^{*}-N M=0$}

The general solution of Eq. (A4) as given in Eq. (A8) can be rewritten in complex form as

$$
A\left(\xi, \xi^{\prime}\right)=M\left(\xi, \xi^{\prime}\right) A\left(-\xi^{\prime}\right)+N\left(\xi, \xi^{\prime}\right) A^{*}\left(-\xi^{\prime}\right) .
$$

Substituting Eq. (B1) into Eq. (A4), we obtain

$$
\begin{aligned}
& \frac{d}{d \xi} M\left(\xi, \xi^{\prime}\right)=T^{\prime} N^{*}\left(\xi, \xi^{\prime}\right), \\
& \frac{d}{d \xi} N\left(\xi, \xi^{\prime}\right)=T^{\prime} M^{*}\left(\xi, \xi^{\prime}\right) .
\end{aligned}
$$

The initial conditions are

$$
\begin{aligned}
& M\left(\xi^{\prime}, \xi^{\prime}\right)=I, \\
& N\left(\xi^{\prime}, \xi^{\prime}\right)=0 .
\end{aligned}
$$

Using Eqs. (B2) and (B3), and the fact that $T^{\prime}$ is symmetric, we obtain

$$
\frac{d}{d \xi}\left(M^{T} M^{*}-N^{\dagger} N\right)=0,
$$

$$
\frac{d}{d \xi}\left(M^{T} N^{*}-N^{\dagger} M\right)=0
$$

Using Eqs. (B4), (B5), (B6), and (B7), we end up with

$$
\begin{aligned}
& M^{T} M^{*}-N^{\dagger} N=I, \\
& M^{T} N^{*}-N^{\dagger} M=0 .
\end{aligned}
$$

When the pump focus is at the center of the interaction, the results of Appendix A can be used to write Eqs. (B8) and (B9) as

$$
\begin{aligned}
& M M^{*}-N N=I, \\
& M N^{*}-N M=0 .
\end{aligned}
$$


[1] D. F. Walls, Nature (London) 390, 141 (1983).

[2] J. Opt. Soc. Am. B 4 (10) (1987), special issue on squeezed states of light, edited by H. J. Kimble and D. F. Walls.

[3] H. J. Kimble, Phys. Rep. 219, 227 (1992).

[4] M. E. Anderson, D. F. McAlister, M. G. Raymer, and M. C. Gupta, J. Opt. Soc. Am. B 14, 3180 (1997).

[5] L. A. Wu, H. J. Kimble, J. L. Hall, and H. Wu, Phys. Rev. Lett. 57, 2520 (1986).

[6] A. La Porta and R. E. Slusher, Phys. Rev. A 44, 2013 (1991).
[7] R. R. Tucci, Int. J. Mod. Phys. B 7, 4403 (1993).

[8] S. K. Choi, R. D. Li, C. Kim, and P. Kumar, J. Opt. Soc. Am. B 14, 1564 (1997).

[9] C. M. Caves, Phys. Rev. D 26, 1817 (1982).

[10] O. Aytür and P. Kumar, Opt. Lett. 17, 529 (1992).

[11] C. Kim and P. Kumar, Phys. Rev. Lett. 73, 1605 (1994).

[12] H. P. Yuen and V. W. S. Chan, Opt. Lett. 8, 177 (1983).

[13] J. H. Shapiro, IEEE J. Quantum Electron. 21, 237 (1985). 